\title{
Regarding "Computer-Assisted Detection of Cerebral Aneurysms in MR Angiography in a Routine Image-Reading Environment: Effects on Diagnosis by Radiologists"
}

W e thank Miki et $\mathrm{al}^{1}$ for presenting their results on using computer-assisted detection (CAD) with MR angiography to identify cerebral aneurysms. Their article raises some pertinent questions and concerns.

With the final consensus by 2 radiologists as the reference standard, the sensitivity for MRA detection of aneurysms was $64 \%$ and $69 \%$, respectively, and went up to $69 \%$ with CAD. Such poor sensitivity raises the question of the utility of MRA as a screening test, despite all these studies having been performed on a 3T scanner. Should $<70 \%$ sensitivity be considered adequate for a screening test of the whole population?

To assess the utility of CAD, one has to look at the aneurysms supposedly missed by radiologists and picked up by CAD. In 124/ 203 cases, the radiologist did not see the aneurysm even after CAD pointed it out. In 90 of these cases, the radiologists did not change their diagnoses, even after CAD, but changed their minds when consensus was reached with another person.

CAD failed to detect 37/203 aneurysms as the top 3 lesion candidates, including 2 aneurysms measuring $\geq 5 \mathrm{~mm}$.

Most of the aneurysms missed by CAD were $\leq 4 \mathrm{~mm}$. As expected, these are also the ones missed frequently by the radiologists. As per the study, CAD detected 24 additional aneurysms, only 1 of which was $>4 \mathrm{~mm}$.

The authors report that the median time required for reviewing the $\mathrm{CAD}$ results and giving feedback was 16 seconds. However, in a routine clinical setting, it is difficult to imagine that incorporating CAD would have such little impact on workflow and reading time, especially when the finding considered suspi- cious by CAD is frequently not considered positive by the radiologist subsequently.

Using consensus as the reference standard also raises an important question based on the frequency at which opinions were changed after consulting with another radiologist, creating subjectivity on the results.

It would be interesting to see whether the authors could share the outcomes/differences in patient management on the basis of these results. Very small aneurysms have a very low reported rupture rate. ${ }^{2}$ The prevalence of a $7 \%$ incidental detection rate, with most being small aneurysms, begs the question of how to manage these small incidental lesions and the utility of such a screening program.

The authors' conclusions that CAD is feasible and can add value is open to interpretation, given the data in this study. A screening program with such inadequate sensitivity, high subjectivity, and unclear outcome needs further clarification and consideration.

\section{REFERENCES}

1. Miki S, Hayashi N, Masutani Y, et al. Computer-assisted detection of cerebral aneurysms in MR angiography in a routine image-reading environment: effects on diagnosis by radiologists. AJNR Am J Neuroradiol 2016;37:1038-43 CrossRef Medline

2. Murayama $Y$, Takao H, Ishibashi T, et al. Risk analysis of unruptured intracranial aneurysms: prospective 10-year cohort study. Stroke 2016;47:365-71 CrossRef Medline

(iD) X. Wu (D) V.B. Kalra (1)D. Durand

(1) R.C. Liu

(D)A. Malhotra

Department of Radiology and Biomedical Imaging Yale School of Medicine New Haven, Connecticut 